
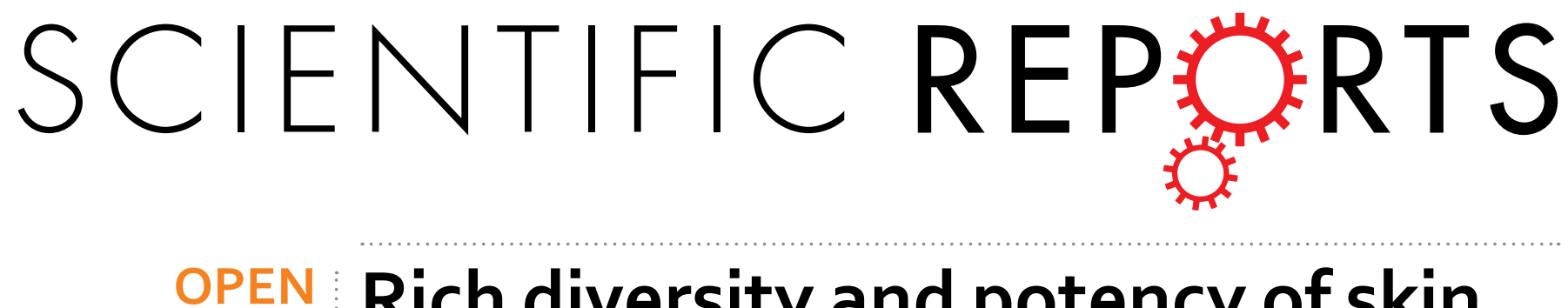

\title{
Rich diversity and potency of skin antioxidant peptides revealed a novel molecular basis for high- \\ altitude adaptation of amphibians
}

Received: 15 July 2015

Accepted: 18 December 2015

Published: 27 January 2016
Xinwang Yang ${ }^{1,3, *}$, Ying Wang ${ }^{2, *}$, Yue Zhang $^{1}$, Wen-Hui Lee ${ }^{1}$ \& Yun Zhang ${ }^{1}$

Elucidating the mechanisms of high-altitude adaptation is an important research area in modern biology. To date, however, knowledge has been limited to the genetic mechanisms of adaptation to the lower oxygen and temperature levels prevalent at high altitudes, with adaptation to UV radiation largely neglected. Furthermore, few proteomic or peptidomic analyses of these factors have been performed. In this study, the molecular adaptation of high-altitude Odorrana andersonii and cavernicolous $O$. wuchuanensis to elevated UV radiation was investigated. Compared with $O$. wuchuanensis, $O$. andersonii exhibited greater diversity and free radical scavenging potentiality of skin antioxidant peptides to cope with UV radiation. This implied that $O$. andersonii evolved a much more complicated and powerful skin antioxidant peptide system to survive high-altitude UV levels. Our results provided valuable peptidomic clues for understanding the novel molecular basis for adaptation to high elevation habitats.

All organisms adapt to specific environments to ensure survival. Elucidating the molecular basis of adaptation is a fundamental and long-standing goal of modern evolutionary biology ${ }^{1,2}$. High-elevation $(>2500 \mathrm{~m})$ environments impose severe physiological challenges on organisms, particularly in regards to the reduced oxygen level, low temperature and elevated ultraviolet (UV) radiation ${ }^{3,4}$. Animals that have survived thousands of years in highlands have evolved adaptive mechanisms during their evolutionary history to cope with these harsh environmental stresses. The mechanisms of animal adaptation to high-altitude environments have been of considerable interest in recent years, and many genetic studies have been conducted on specific candidate genes, such as hemoglobin-related and mitochondrial genes ${ }^{5-7}$. Recent analysis of genome-wide variations among species and populations living in different altitudinal environments, including animal populations of $\mathrm{yak}^{8}$, Tibetan antelope ${ }^{9-11}$, snow leopard ${ }^{12}$ and wild boar ${ }^{13}$, human populations in the Andes ${ }^{10,14}$ and Ethiopia ${ }^{15,16}$, have begun to shed light on the genetic basis of many adaptive responses to low temperature and reduced oxygen at high altitudes. However, elevated UV radiation remains a neglected area of study. To date, genome-wide studies have only investigated endothermic species, with little information available on ectothermic species. Given the large physiological differences between such species, different mechanisms for adaptation to high-elevation environments are likely to have evolved. For example, instead of maintaining high metabolism for temperature stability, ectothermic species may lower their metabolic rate to cope with low external temperatures ${ }^{4}$. Current research on the molecular basis of high-altitude adaptation has primarily relied on genomic analysis, with comparative proteomic analyses, which are essential for understanding how animals adapt to high-elevation environments, still in their infancy. As yet, only a few related works have been conducted on plants ${ }^{17}$.

Amphibians, the first group of animals to form a link between terrestrial and aquatic environments, are widely distributed in a great variety of habitats worldwide. Amphibians living in high-altitude environments

\footnotetext{
${ }^{1}$ Key Laboratory of Animal Models and Human Disease Mechanisms, Chinese Academy of Sciences \& Yunnan Province, Kunming Institute of Zoology, Chinese Academy of Sciences, Kunming 650223, Yunnan, China. ${ }^{2}$ Key Laboratory of Chemistry in Ethnic Medicine Resource, State Ethnic Affairs Commission \& Ministry of Education, School of Ethnic Medicine, Yunnan Minzu University, Kunming 650500, China. ${ }^{3}$ Department of Anatomy and Histology \& Embryology, Faculty of Basic Medical Science, Kunming Medical University, Kunming 650500, China. *These authors contributed equally to this work. Correspondence and requests for materials should be addressed to Y.Z. (email: zhangy@mail.kiz.ac.cn)
} 


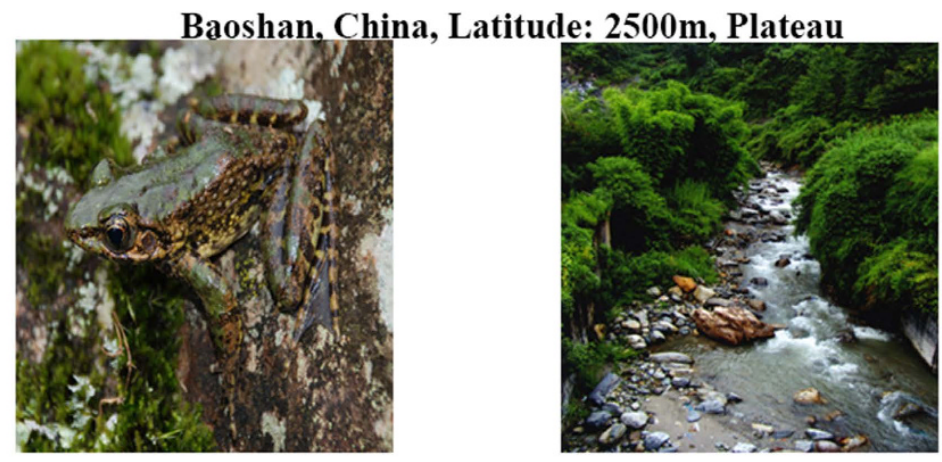

Wuchuan, China, Latitude:800 m, Cave
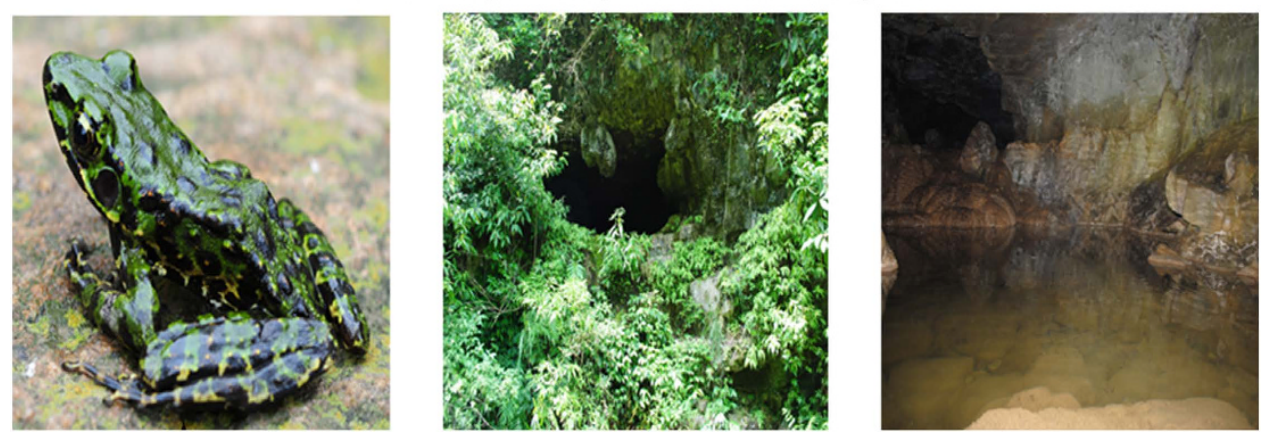

Figure 1. Distribution areas of $O$. andersonii and $O$. wuchuanensis. Odorrana andersonii is widely distributed on the Yun-Gui Plateau and was collected in Baoshan city in Yunnan Province, China. Odorrana wuchuanensis is only distributed in a few mountain caves in Guizhou Province.

have evolved many behavioral and physiological adaptations to low temperatures and reduced oxygen at high altitudes $^{18-21}$. For example, the adaptive significance of the alpha-chain chloride-binding sites of hemoglobin in response to low oxygen has been reported ${ }^{22}$. In addition, recent transcriptomic analysis of two amphibian species revealed that a set of candidate genes, including genes associated with oxygen binding, UV radiation response and free radical injury repair, may be related to high-elevation environments ${ }^{23}$. However, high-altitude adaptation in amphibians remains to be fully elucidated.

Skin is exposed to both endogenous and exogenous sources of oxidative stress and has developed various mechanisms to cope with increased oxidation ${ }^{24,25}$. One of the most important strategies is the modulation of the antioxidant system, which can be classified into two major groups: gene-encoded enzymes and non-gene-encoded low-molecular-weight antioxidants. The first group includes superoxide dismutase (SOD), catalase and peroxidase. The second group includes molecules such as glutathione (GSH) and vitamins $\mathrm{C}$ and $\mathrm{E}^{24,26}$. Radiation injury is considered one of the most significant non-biological stresses for amphibians because their skin is frequently exposed to a variety of harsh conditions; consequently, amphibian skin is endowed with an excellent chemical defense system composed of bioactive peptides, including a novel type of antioxidant peptides (AOPs) that was identified recently and characterized by gene-coded low-molecular weight peptides ${ }^{27-29}$. Amphibians living in plateau environments with strong UV radiation and a long duration of sunshine are at greater risk of radiation injury, and the identity and potential role of antioxidant peptides (AOPs) in high-altitude adaptation remains unclear.

Odorrana andersonii and O. wuchuanensis provide an excellent model system to study the adaptation of amphibian skin to high-altitude habitats. Odorrana andersonii lives in plateau areas (approximately $2500 \mathrm{~m}$ ) of the Yunnan Province in China, which are characterized by a long duration of sunshine and strong UV radiation. Odorrana wuchuanensis is only distributed in a small number of caves (at an altitude of $800 \mathrm{~m}$ ) in the Guizhou Province of China and encounter no light during their entire life cycle. Thus, we hypothesized that the AOPs of these two odorous frogs differ in amounts, structural characteristics and activities. Our results confirmed this hypothesis and provided valuable peptidomic clues for understanding the molecular basis of high-altitude adaptation in amphibians.

\section{Results}

Inducement of antioxidant activity of amphibian skin secretions by UV radiation. As shown in Fig. 1, O. andersonii is widely distributed on the Yun-Gui Plateau in southwest China, whereas O. wuchuanensis is only distributed in a few mountain caves in Guizhou Province. To simulate the UV-radiated conditions of $O$. andersonii habitat, we exposed the skin of all frogs to UVB radiation $\left(1600 \mu \mathrm{W} / \mathrm{cm}^{2}\right)$ for $9 \mathrm{~h}$. Compared with the dark environment, the antioxidant activities of the skin secretions from both species increased to different degrees following UVB radiation. The antioxidant activity of $O$. andersonii skin secretions increased after $1 \mathrm{~h}$ of 


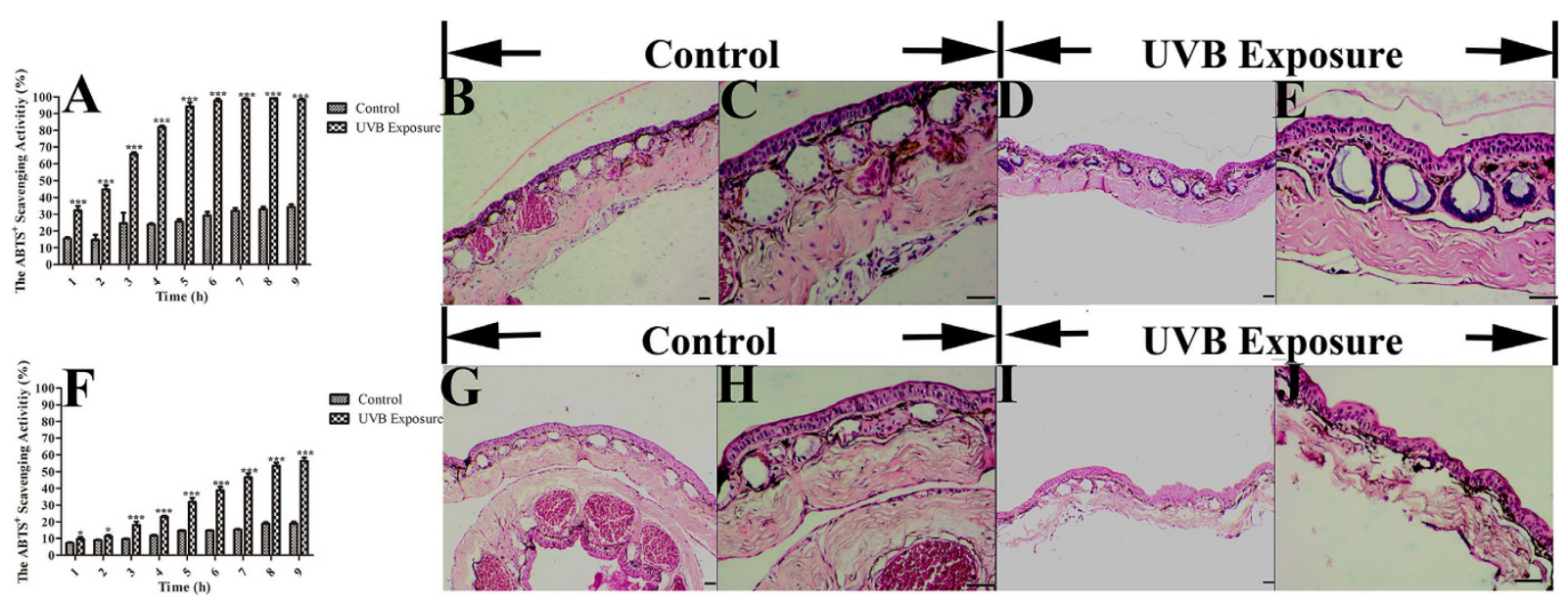

Figure 2. Response of $O$. andersonii and $O$. wuchuanensis skins to UVB radiation. Skins of $O$. andersonii and $O$. wuchuanensis were exposed to UVB radiation at a dose of $1600 \mu \mathrm{W} / \mathrm{cm}^{2}$ for $9 \mathrm{~h}$. (A,F) show antioxidant activities in $O$. andersonii and $O$. wuchuanensis skin secretions induced by UVB exposure, respectively. The antioxidant activities were tested every $1 \mathrm{~h}$. (B,C,G,H) show normal skin structures of $O$. andersonii and O. wuchuanensis, respectively. $(\mathbf{E}, \mathbf{F}, \mathbf{I}, \mathbf{J})$ show effects of $\mathrm{UVB}$ radiation on the skin structures of $O$. andersonii and $O$. wuchuanensis, respectively. ${ }^{\star} \mathrm{P}<0.01,{ }^{\star *} \mathrm{P}<0.001$. Bar $=250 \mu \mathrm{m} .(\mathbf{B}, \mathbf{D}, \mathbf{G}, \mathbf{I})$ are at $40 \times,(\mathbf{C}, \mathbf{E}, \mathbf{H}, \mathbf{J})$ are at $100 \times$.

UVB exposure and reached a maximum of $\sim 99 \%$ after 5 h (Fig. 2A). For O. wuchuanensis, however, antioxidant activity did not exhibit an obvious increase until $3 \mathrm{~h}$ of UVB exposure and reached a maximum of only $\sim 50 \%$ after $9 \mathrm{~h}$ (Fig. 2F). Thus, the antioxidant activities of both amphibian skin secretions were remarkably inducible by UV radiation, but the high-altitude $O$. andersonii skin secretions exhibited more potential antioxidant activates than cavernicolous $O$. wuchuanensis. These differences might be attributable to differences in the secretion, concentration or potency of the antioxidant substances induced by UVB radiation.

Potential tolerance of $O$. andersonii and $O$. wuchuanensis skins to UV radiation. To characterize the damage to frog skin induced by UVB radiation, we performed HE staining to examine alterations in general frog skin morphology. Control skins not exposed to UVB radiation displayed a compact and stratified structure with normal epidermis, glands, dermis and blood vessels, with no evidence of inflammation or other damage (Fig. 2B,C,G,H). After direct UVB radiation, the skins of $O$. andersonii exhibited no obvious change (Fig. 2B,C), whereas the skins of $O$. wuchuanensis exhibited obvious tissue damage, including acute epidermal necrosis with inflammatory infiltrates in the superficial and deep dermis and destruction of glands (Fig. 2G,H). Antioxidants usually protect skin from impairment caused by UV radiation, and the different levels of damage to the skin of $O$. andersonii and $O$. wuchuanensis might be attributable to differences in the antioxidant activities of skin secretions. We speculated that the strong antioxidant activity of $O$. andersonii secretions protected their skin against damage induced by UV radiation.

AOP diversity in 0 . andersonii and 0 . wuchuanensis skin secretions. Under normal conditions without stimulation, the skins of both $O$. andersonii and $O$. wuchuanensis secreted few peptides or proteins (Fig. 3A,B). Following exposure to UVB radiation, however, an increase in peptide secretions was observed (Fig. 3C,D). Although molecular weights of these UVB-induced peptides were less than $4000 \mathrm{Da}$, remarkable differences in diversity were observed via mass spectrometry (MS) (Fig. 3E,F), which revealed that O. andersonii secreted a greater diversity of peptides (more than 100 molecules) than that of $O$. wuchuanensis (approximately 40 molecules). This difference in diversity was confirmed by initial HPLC analysis (Fig. 3G,H), which revealed more than 110 compounds in $O$. andersonii skin secretions but only 70 compounds in O. wuchuanensis skin secretions. The exact structures and functions of these peptides have not been investigated, but they likely play an important role in the scavenging of free radicals. Thus, in the present study, we characterized and compared peptides with the ability to scavenge free radicals.

Identical purification procedures were used to identify AOPs in skin secretions induced by UVB exposure. After the first RP-HPLC purification step, 14 fractions with antioxidant activities were separated from high-altitude O. andersonii (Fig. 3G), but only 5 fractions were purified from cavernicolous O. wuchuanensis (Fig. $3 \mathrm{H})$. Further purification enabled the isolation of 32 and 5 fractions with antioxidant activity and sufficient purity from the skin secretions of O. andersonii (Fig. S1) and O. wuchuanensis (Fig. S2), respectively. The complete amino acid sequences of these AOPs were presented in Fig. 4. After screening the skin cDNA library, another 10 molecules showing high sequence similarity were identified from O. andersonii AOPs, and were thus assumed to be AOPs. In total, 42 and 5 AOPs were identified by a combination of peptidomic and genomic methods from the skin secretions of $O$. andersonii and $O$. wuchuanensis, respectively (Fig. 4). The GenBank accession numbers assigned to the cDNA sequences encoding these AOPs are JX507085-JX507126.

Based on sequence similarity provided by BLASTp searches in NCBI, these identified AOPs from O. andersonii were grouped into 26 families named as Andersonin-AOP1 to Andersonin-AOP26 according to peptides' 

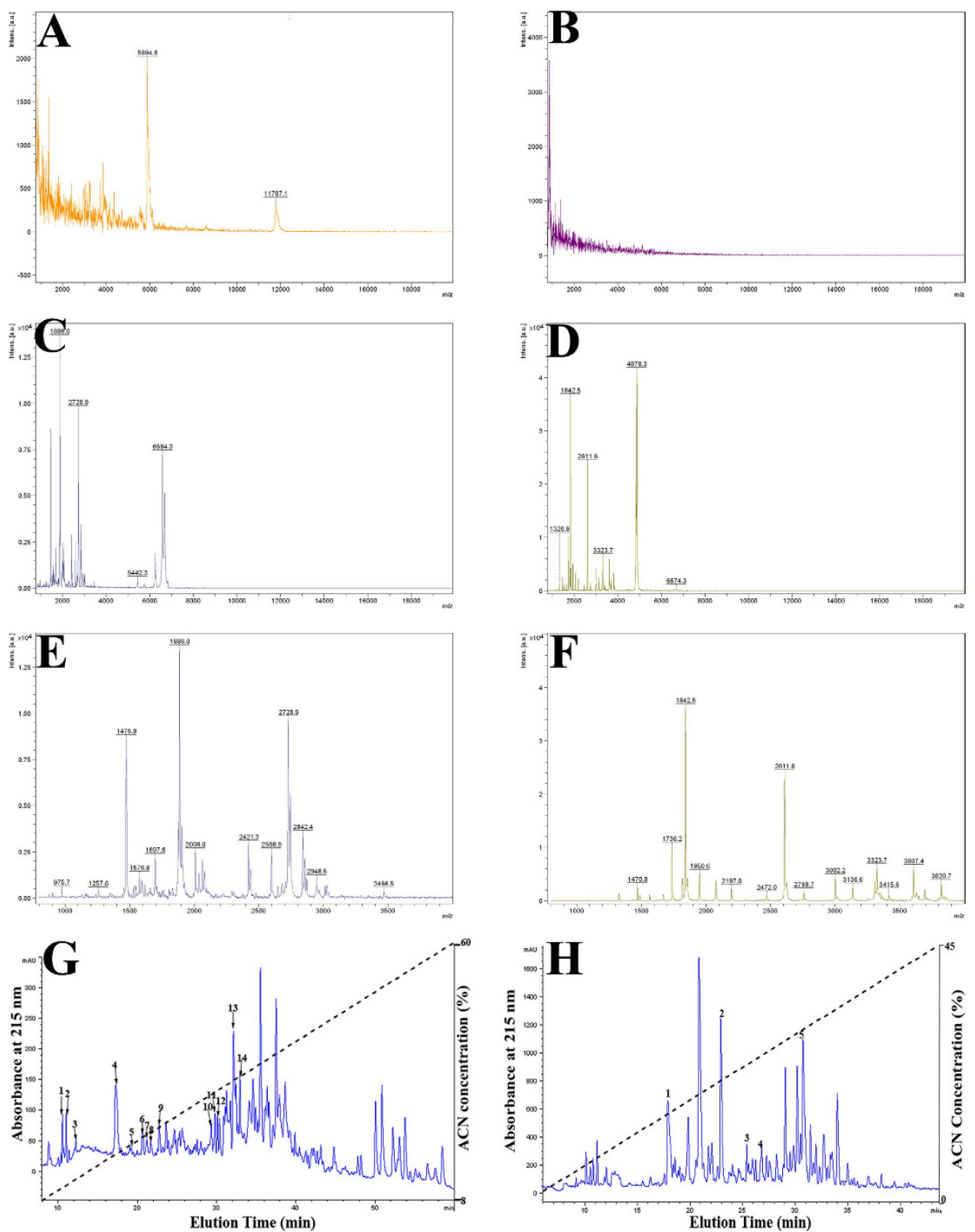

Figure 3. Overview of peptides induced by UV radiation. MS and HPLC analyses showed that UV radiation induced the secretion of many peptides compared with the controls. Left $O$. andersonii and right O. wuchuanensis. (A) MS analysis of O. andersonii skin secretions without UV exposure. (B) MS analysis of O. wuchuanensis skin secretions without UV exposure. (C) MS analysis of O. andersonii skin secretions with UV exposure, molecular weight range 800-20000 Da. (D) MS analysis of O. wuchuanensis skin secretions with UV exposure, molecular weight range 800-20000 Da. (E) MS analysis of O. andersonii skin secretions with UV exposure, molecular weight range 800-4000 Da. (F) MS analysis of O. wuchuanensis skin secretions with UV exposure, molecular weight range 800-4000 Da. (G) First RP-HPLC purification step of AOPs from skin secretions of $O$. andersonii. Skin secretions were applied to a $\mathrm{C}_{18}$ RP-HPLC column pre-equilibrated with $0.1 \%$ $(\mathrm{v} / \mathrm{v})$ TFA in water, and elution was achieved by a linear gradient $(0-100 \%$ in $100 \mathrm{~min})$ of $0.1 \%(\mathrm{v} / \mathrm{v})$ TFA in $\mathrm{ACN}$ at a flow rate of $1 \mathrm{~mL} / \mathrm{min}$ and was monitored at $215 \mathrm{~nm}$. Fourteen peaks with $\mathrm{ABTS}^{+}$scavenging activity were further purified. (H) First RP-HPLC purification step of AOPs from skin secretions of $O$. wuchuanensis. Skin secretions were applied to a $\mathrm{C}_{18}$ RP-HPLC column pre-equilibrated with $0.1 \%$ (v/v) TFA in water, and elution was achieved by a linear gradient $(0-100 \%$ in $100 \mathrm{~min})$ of $0.1 \%(\mathrm{v} / \mathrm{v})$ TFA in ACN at a flow rate of 1 $\mathrm{ml} / \mathrm{min}$ and was monitored at $215 \mathrm{~nm}$. Five peaks with $\mathrm{ABTS}^{+}$scavenging activity were further purified.

length. Similarly, the AOPs from O. wuchuanensis were named as Wuchuanin-AOP1 to Wuchuanin-AOP5 (Fig. 4). Among these AOPs, we have previously reported Andersonin-AOP12, -AOP16 and -AOP20 and identified another eight peptides, including four from O. andersonii (Odorranain-A-OA12, Andersonin-G1, -H3 and $-\mathrm{R} 1$ ) and four from O. wuchuanensis (Wuchuanin-A1, -C1, -D1 and -E1), with obvious antioxidant activity ${ }^{30}$. In 


\begin{tabular}{|c|c|c|}
\hline & eptide & ite Mature peptide \\
\hline Andersonin-AOP1 & KSLLLIFFLGTTNLSLCQEETNAEEERRDDPNEMDAEGT & \\
\hline Andersonin-AOP2 & METLKKTLLLLFFLGI INLSLCEEEERNAEEEERRDDPDVRDVEVE & KRSLSCFLSFTR \\
\hline Andersonin-AOP3 & MFTLKKSLLFLFFIGIVSSSPYRGKRENGEEEAIEDI & KRQLFLQGSPQC \\
\hline Andersonin-AOP $4 a$ & MFTLKKPLLLLFFLGI ISLSFCEQERDADEEDGGEVTEEEV & KREMHFRLCAHF \\
\hline Andersonin-AOP $4 \mathrm{~b}$ & MFTLKKPLLLLFFLGIISLSFCEQERDADEEDGGEVTEEEV & KRFMHFRLFAAF \\
\hline Andersonin-AOP5a & MFTLKKPLLLLFFLGI ISLSFCEQERDADEEDGGEVTEEEV & KRFFSLCGLSGM \\
\hline Andersonin-AOP5b & MFTLKKPLLLLFFLGI ISLSFCEQERDADEEDGGEVTEEEV & KRFFMLCGLSGM \\
\hline Andersonin-AOP6 & MFTLKKSLLLLFFLGIVSLSLCEEEKRDADEEGNEENEAEAKVKDI & KRRLFKCYKPDS \\
\hline Andersonin-AOP7 & MFTMKKSMLLLFFLGTISLSLCEEERNADEEERRDEEVAKMEEI & KRECFRYPNGMLAR \\
\hline Andersonin-AOP8a & MFTMKKSLLLLFFLGTISLPLCEQERDAEEEGSENGAEDIKL & NRLMYGLGSPDSRCN \\
\hline Andersonin-AOP8b & MFTMKKSLLLLFFLGT ISLPLCEQERDAEEEGSENGAEDIKL & NRLKFGMFHKMARCN \\
\hline Andersonin-AOP8C & MFTLKKSLLLLFFLGTISLPLCEQERDAEEEGSENGAEDIKL & KRALFLLHSPDSRCN \\
\hline Andersonin-AOP8d & MFTLKKSLLLLFFLGTISLPLCEQERDAEEEGSENGAEDIKL & KRALFLLGSPDSRCN \\
\hline Andersonin-AOP8e & MFTMKKSLLLLFFLGTISLPLCEQERDAEEEGSENGAEDIKL & NRNSLSFFLSFTRCQ \\
\hline Andersonin-AOP8 $f$ & MFTMKKSLLLLFFLGTISLPLCEQERDAEEEGSENGAEDIKL & NRNSLSFFLSFTRCN \\
\hline Andersonin-AOP9 & MFTMKKPLLLIVLIGI ISLSLCEQERAADEDEGSEM & KRLKGFEMGMDMKRT \\
\hline Andersonin-AOP10a & MFPLKKTLLLLFFFGIVPLAFCEPGRDSDEEENEGHGAEANFENL & KR SYLNSLSCFLSFT \\
\hline Andersonin-AOP10b & MFPLKKTLLLLFFFGIVPLAFCEPGRDSDEEENEGHGAEANFENL & KRSMLNSLSCELSFT \\
\hline Andersonin-AOP1la & MFTLKKSLLFLFFLGTINLSLCQEETNAEEERRDEEVAKMEE I & KRGMGYMMLCGLSGM \\
\hline Andersonin-AOP11b & MFTLKKSLLFLFFLGTINLSLCQEETNAEEERRDEEVAKMEEI & KRGMGFMMLCGLSGM \\
\hline Andersonin-AOP11c & MFTLKKSLLFLFFLGTINLSLCQEETNAEEERRDEEVAKMEEI & KRGMGFFKMCGLSGL \\
\hline Andersonin-AOP12* & MFTLKKPLLLLFFLGTINLSECQDETNAEEERRDEEVAKMEET & KRTSRCIFYRRKKCS \\
\hline Andersonin-AOP13 & MFTLKKSLLLLFFLGTISLSLCEEEKRYANGEAYEENVRDANIE & KRAPDRPRKFCGILG \\
\hline Andersonin-AOP14a & \multicolumn{2}{|c|}{ MFTLKKSLLLLFFLGIVSLSLCEQERDADEEGNEEYRGEAKVENIKRVADMMLKRTGKDMAGCFKFGE } \\
\hline Andersonin-AOP14b & \multicolumn{2}{|c|}{ MFTLKKSLLLLFFLGIVSLSLCEQERDADEEGNEEYRGEAKVENIKRVADMMLKR GGKDMAGCFKFGE } \\
\hline Andersonin-AOP14c & \multicolumn{2}{|c|}{ MFTLKKSLLLLFFLGIVSLSLCEQERDADEEGNEEYRGEAKVENIKRVADMMLKRLGKDMAGCFKFGE } \\
\hline Andersonin-AOP14d & \multicolumn{2}{|c|}{ MFTLKKSLLLLFFLGIVSLSLCEQERDADEEGNEEYRGEAKVENIKRVADMMLKRTGKHMAGCFKFGES } \\
\hline Andersonin-AOP14e & \multicolumn{2}{|c|}{ MFTLKKSLLLIFFLGIVSLSLCEQERDADEEGNEEYRGEAKVENIKRVADMMLKRTGKDMAGCFKFGES } \\
\hline Andersonin-AOP15 & MFFLKKSLLSFSFLGPSSYLFVRRDNTEEERRDEEEVK & KRAYMKQHMYCAASFF \\
\hline Andersonin-AOP16* & MFTLKKSLLLLFFLGTISLSLCEQERDAEEEEGSENGAEDIKL & NRVVKCSYRQGSPDSR \\
\hline Andersonin-AOP17 & MFTMKKSLLLLFFLGI ISLSLCEEERDANEEEENEENGGETKVVEG & KRVTPPWARIYYGCAKA \\
\hline Andersonin-AOP18 & MFTLKKTLSLLFFLGTISLSLCEEERDADEEENEVSGYAANVNI & KREMFFCYFPDSRGFQMH \\
\hline Andersonin-AOP19a & MFTMKKSLLLLFFLGTINLSLCEEERGADEEDGGEAKLEDI & KRGAGFWKMGKYGQKRRD \\
\hline Andersonin-AOP19b & MFTMKKSLLLLFFLGTINLSLCEEERGADEEDGGEAKLEDI & KRGAGFWKMGKLGQKRRD \\
\hline Andersonin-AOP20* & MFTLKKPLLLLFFLGTISLSLCQDETNAEEERRDEEVAKMEEI & KRENMFNIKSSVESDSFWG \\
\hline Andersonin-AOP21a & MFTLKKFLLLLFFLGTISLSLCEEEKRYANGEADEENVRDANIE & KRMMLKRGGKDMAGCFKFGE \\
\hline Andersonin-AOP21b & MFTLKKSLLLLFFLGTISLSLCEEEKRYANGEAYEENVRDENIE & KRMMLKRLGKDMAGCFKFGE \\
\hline Andersonin-AOP22 & MFTLKKPLLLLFFLGTISLSLCQDETNAEEERRDEEVAKMEEI & KR GLFTLIKGAYKLDAPTVACN \\
\hline Andersonin-AOP23 & MFTLKKSLLFLFFLGTINLSLCQEETNAEEERRDEEVAKMEEI & KRGLFSMILGVGKKTLCGLSGLW \\
\hline Andersonin-AOP24 & MFTLKKSLLLLFFLGT INLSLCQEETNAEEERRDEEVAKMEE I & KRGIFPLILGLGKKIYVDFLGCAKA \\
\hline Andersonin-AOP25* & MFTLKKSLLLLFFKGIVSSSPCFRKRDADEEGNEENGGEAKMEDI & KRATALGIPPRGFLPIVNKKFKDIILC \\
\hline Andersonin-AOP26 & MFTLKKFLLLLFFFGIVSSSPCFRKRDADEEGNEENGAEAKIEDI & KR IPWKLPATLRPVENPFFSKPLCRNY \\
\hline Wuchuanin-AOP1 & MFTLKKPLLLLFFLGTINLSSCQDERNAEEERRDDSDEMNAEVE & KRDKRPYILRE \\
\hline Wuchuanin-AOP2 & MFTLKKPLLLLFFLGTISLSLCQDETNAEEERRDEEVAKMEEI & KRGFRPTKPVPF \\
\hline Wuchuanin-AOP3 & MFTLKKPLLLLFFLGAISLSFCEQERDAEEEGNEENRVEVQ & KRGLIVRGLSGLR \\
\hline Wuchuanin-AOP4 & MFTMKKSLLLLFFLGTISLSFCEQERDAEDEEENGGEAKVEEI & KRAVNIPFKVHFR \\
\hline Wuchuanin-AOP5 & MFTLKKSLLLLFFLGAISLSLCODETNAEEERRDEEVAKMEEI & KRTVWGERPSKPPSGYR \\
\hline
\end{tabular}

Figure 4. Structural diversity of AOPs. A total of 42 AOPs classified into 26 families were identified from $\mathrm{O}$. andersonii. By contrary, only 5 AOPs were identified from $\mathrm{O}$. wuchuanensis. All precursors of these identified AOPs shared a highly conserved motif, which was divided into four parts from the N-terminus to the $\mathrm{C}$-terminus. The first part was an $\mathrm{N}$-terminal hydrophobic signal peptide of about 22 residues in length, followed by an acidic segment that differed in length, ranging from 16-25 residues. The third part was a typical enzyme cleavage site 'Lys-Arg', sometimes mutated to 'Val-Arg', with the final part a C-terminal section of the mature peptide. The mature peptides confirmed by Edman degradation are underlined. ' ${ }^{\prime}$ 'indicates that this peptide was identified and reported in our previous study ${ }^{30}$.

all, 46 AOPs classified into 30 different families were identified from high-altitude O. andersonii, whereas only nine AOPs grouped into 9 different families were recognized from cavernicolous $O$. wuchuanensis. These results indicated that the diversity of skin AOPs in high-altitude $O$. andersonii skins was much richer than that in cavernicolous $O$. wuchuanensis.

Structural characteristics of AOPs from skins of $\boldsymbol{O}$. andersonii and $\boldsymbol{O}$. wuchuanensis. As shown in Fig. 4, all precursors of the identified AOPs shared a highly conserved motif that was divided into 4 parts from the $\mathrm{N}$-terminus to the C-terminus. The first part was an $\mathrm{N}$-terminal hydrophobic signal peptide approximately 22 residues in length, followed by an acidic segment that differed in length from 16-25 residues. The third part was a typical 'Lys-Arg' enzyme cleavage site, which was occasionally mutated to 'Val-Arg'. The final part corresponded to the C-terminal section of the mature peptide.

As listed in Table S1, the molecular weights of the 37 AOPs determined by MS matched well with their theoretical molecular weights, indicating a lack of post-translational modification. Most of the AOP families contained only one member; however, some families exhibited great diversity, such as Andersonin-AOP8s and -AOP14s, which included six and five members, respectively (Fig. 3). The length of the AOPs also exhibited considerable 


\begin{tabular}{|c|c|c|c|}
\hline \multirow[b]{2}{*}{ Samples } & \multicolumn{3}{|c|}{ Free radical scavenging activity (\%) } \\
\hline & ABTS $^{+}$ & Figure & DPPH \\
\hline $\mathrm{H}_{2} \mathrm{O}$ & ND & & ND \\
\hline Vitamin C & $98.52 \pm 1.34$ & & $93.97 \pm 1.49$ \\
\hline Andersonin-AOP1 & $99.28 \pm 0.56$ & $5 \mathrm{~A}$ & ND \\
\hline Andersonin-AOP2 & $98.95 \pm 1.00$ & S3 & $89.39 \pm 2.31$ \\
\hline Andersonin-AOP3 & $98.98 \pm 0.95$ & $\mathrm{~S} 4$ & $64.29 \pm 1.43$ \\
\hline Andersonin-AOP4a & $91.80 \pm 1.79$ & S5 & $69.36 \pm 1.37$ \\
\hline Andersonin-AOP5a & $99.70 \pm 0.44$ & S6 & $71.90 \pm 0.96$ \\
\hline Andersonin-AOP6 & $96.46 \pm 0.69$ & $5 \mathrm{~A}$ & ND \\
\hline Andersonin-AOP7 & $99.07 \pm 0.85$ & S7 & $79.88 \pm 1.29$ \\
\hline Andersonin-AOP8a & $99.61 \pm 0.17$ & S8 & $55.78 \pm 0.98$ \\
\hline Andersonin-AOP8b & $97.61 \pm 1.84$ & S9 & $72.36 \pm 2.01$ \\
\hline Andersonin-AOP8c & $90.61 \pm 1.89$ & $\mathrm{~S} 10$ & $61.11 \pm 1.46$ \\
\hline Andersonin-AOP8e & $99.28 \pm 0.62$ & S11 & $78.21 \pm 2.75$ \\
\hline Andersonin-AOP9 & $51.33 \pm 1.63$ & $\mathrm{~S} 12$ & $11.90 \pm 2.39$ \\
\hline Andersonin-AOP10a & $99.70 \pm 0.44$ & $\mathrm{~S} 13$ & $87.32 \pm 0.35$ \\
\hline Andersonin-AOP11a & $99.47 \pm 0.57$ & $\mathrm{~S} 14$ & $90.23 \pm 4.59$ \\
\hline Andersonin-AOP11c & $94.80 \pm 1.05$ & $\mathrm{~S} 15$ & $65.38 \pm 3.12$ \\
\hline Andersonin-AOP12 & $99.61 \pm 0.44$ & $5 \mathrm{~A}$ & $75.24 \pm 2.52$ \\
\hline Andersonin-AOP13 & $84.69 \pm 0.58$ & S16 & $33.02 \pm 1.45$ \\
\hline Andersonin-AOP14a & $99.64 \pm 0.55$ & S17 & $67.55 \pm 1.58$ \\
\hline Andersonin-AOP14b & $98.31 \pm 1.13$ & S18 & $70.49 \pm 2.51$ \\
\hline Andersonin-AOP14d & $99.30 \pm 1.13$ & S19 & $85.32 \pm 3.78$ \\
\hline Andersonin-AOP15 & $99.04 \pm 1.57$ & $\mathrm{~S} 20$ & $91.01 \pm 4.37$ \\
\hline Andersonin-AOP16 & $98.32 \pm 1.35$ & $\mathrm{~S} 21$ & $86.19 \pm 1.72$ \\
\hline Andersonin-AOP17 & $66.67 \pm 1.81$ & $\mathrm{~S} 22$ & $35.24 \pm 2.08$ \\
\hline Andersonin-AOP18 & $99.16 \pm 1.45$ & $\mathrm{~S} 23$ & $40.23 \pm 2.98$ \\
\hline Andersonin-AOP19a & $60.00 \pm 1.92$ & $5 \mathrm{~A}$ & ND \\
\hline Andersonin-AOP20 & $99.82 \pm 0.09$ & $5 \mathrm{~A}$ & ND \\
\hline Andersonin-AOP21a & $94.69 \pm 0.58$ & S24 & $57.98 \pm 4.92$ \\
\hline Andersonin-AOP22 & $99.25 \pm 1.28$ & S25 & $60.79 \pm 1.53$ \\
\hline Andersonin-AOP23 & $97.95 \pm 2.15$ & S26 & $94.38 \pm 0.50$ \\
\hline Andersonin-AOP24 & $99.96 \pm 0.06$ & $\mathrm{~S} 27$ & ND \\
\hline Andersonin-AOP25 & $95.74 \pm 2.33$ & $\mathrm{~S} 28$ & $77.41 \pm 0.87$ \\
\hline Andersonin-AOP26 & $80.77 \pm 4.36$ & S29 & $41.27 \pm 5.09$ \\
\hline Wuchuanin-AOP1 & $33.12 \pm 1.23$ & S30 & ND \\
\hline Wuchuanin-AOP2 & $13.26 \pm 0.51$ & S31 & ND \\
\hline Wuchuanin-AOP3 & $17.21 \pm 1.01$ & S32 & ND \\
\hline Wuchuanin-AOP4 & $11.89 \pm 0.89$ & S33 & ND \\
\hline Wuchuanin-AOP5 & $92.34 \pm 2.31$ & $5 B$ & ND \\
\hline
\end{tabular}

Table 1. Antioxidant activities of AOPs from the skin of odorous frogs. All AOPs were evaluated at a concentration of $50 \mu \mathrm{M} . \mathrm{H}_{2} \mathrm{O}$ and vitamin $\mathrm{C}$ were used as negative and positive controls, respectively. 'ND' indicates 'Not Detectable. Data are mean values of three independent experiments performed in triplicate.

diversity. Andersonin-AOP1 was the shortest AOP (9 amino acids), whereas Andersonin-AOP25 and -AOP26 were the longest AOP (24 amino acids, respectively).

Based on the number of cysteine residues, the identified AOPs were divided into three subfamilies: Family A, linear AOPs with no cysteine residue; Family B, linear AOPs with one free cysteine residue; and Family C, cyclic AOPs. Among the AOPs from O. andersonii, five and one belonged to Family A and C, respectively, and the remaining 36 belonged to Family B. This indicated that AOPs with one free cysteine were the dominant components of O. andersonii. Conversely, all AOPs from O. wuchuanensis belonged to Family A.

Antioxidant activity of AOPs from 0 . andersonii and 0 . wuchuanensis skins. We also compared the antioxidant activities of AOPs from these two odorous frogs. Most O. andersonii skin AOPs exhibited potential $\mathrm{ABTS}^{+}$scavenging activity (>90\%), except for Andersonin-AOP9, -AOP13, -AOP17 and -AOP19a (Table 1). In comparison, except for Wuchuanin-AOP5, O. wuchuanensis AOPs displayed much weaker antioxidant activity. Moreover, most $O$. andersonii AOPs scavenged the free radical DPPH, whereas no peptides from $O$. wuchuanensis exhibited this activity. 

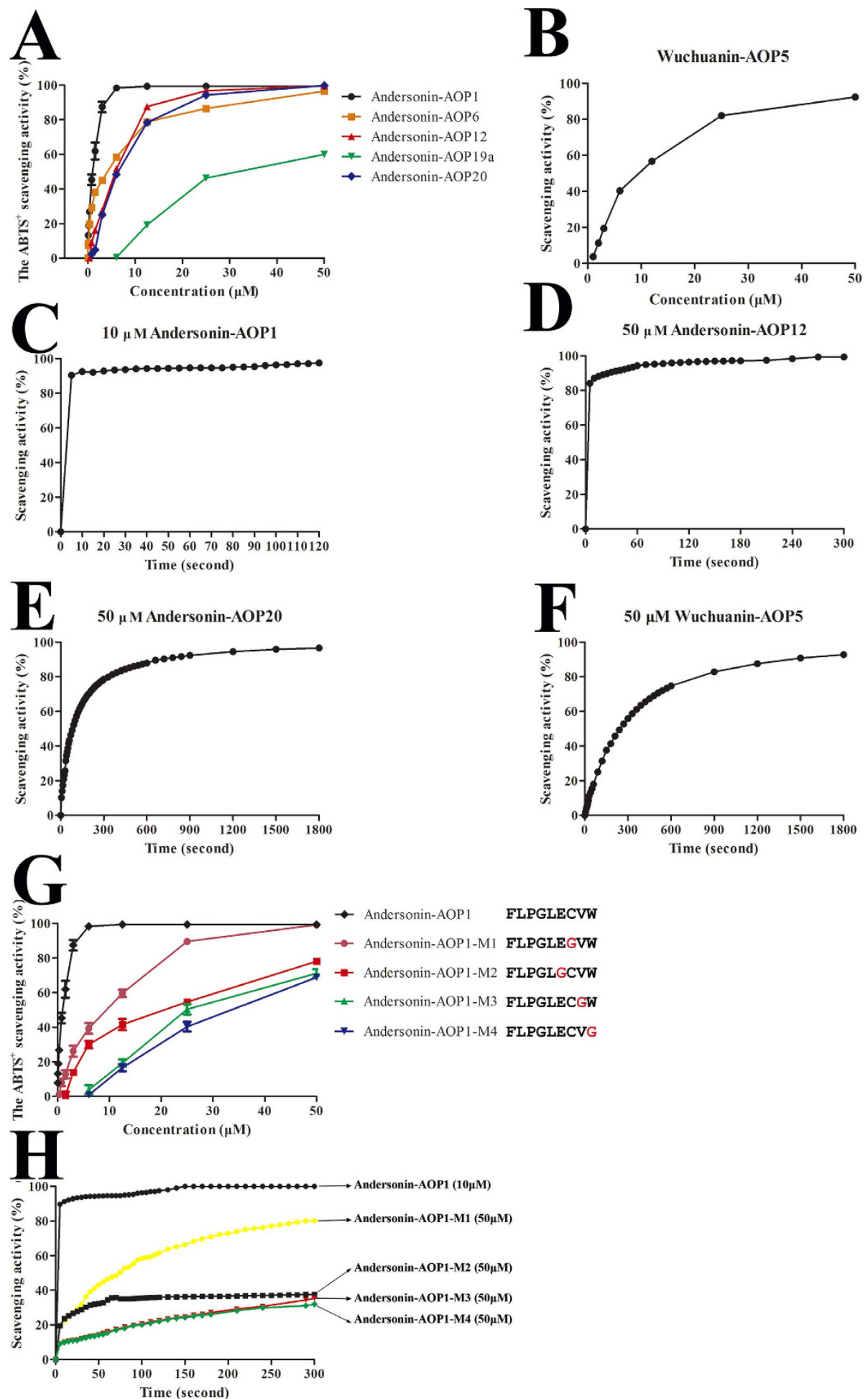

Figure 5. Antioxidant activities of different types of AOPs. (A) Antioxidant activities of AOPs from O. andersonii. Five AOPs representing different molecular types were selected to determine $\mathrm{ABTS}^{+}$scavenging activity. Andersonin-AOP1 with free cysteine residue was the most powerful scavenger, while AndersoninAOP19a, which had no cysteine residue, was the weakest scavenger. (B) Antioxidant activities of AOPs from O. wuchuanensis. Wuchuanin-AOP5 was the most powerful antioxidant; however, its activity was much weaker than that of Andersonin-AOP1. (C-F) Andersonin-AOP1 (representative of AOPs with one free cysteine) and Andersonin-AOP12 (representative of AOPs with one disulfide bridge) exerted high ABTS ${ }^{+}$ scavenging efficiency $(\mathbf{A}, \mathbf{B})$. In contrast, Andersonin-AOP20 and Wuchuanin-AOP5 (representative of AOPs without cysteine) showed comparatively low scavenging efficiency. Data are mean values of three independent experiments performed in triplicate. $(\mathbf{G}, \mathbf{H})$ Four mutants of Andersonin-AOP1 were designed. The replacement of the last four residues resulted in a decrease in $\mathrm{ABTS}^{+}$scavenging ability, and the replacement of free cysteine lead to a notable decrease in $\mathrm{ABTS}^{+}$scavenging efficiency. 
All AOPs scavenged $\mathrm{ABTS}^{+}$in a dose-dependent manner (Figs S3-S33). Andersonin-AOP1 exhibited the most potential $\mathrm{ABTS}^{+}$scavenging activity and scavenged $99.280 \pm 0.562 \%$ of $\mathrm{ABTS}^{+}$at a concentration of $3 \mu \mathrm{M}$ $\left(\mathrm{n}=3\right.$, Fig. 5A). By contrast, Wuchuanin-AOP5, which exhibited the greatest $\mathrm{ABTS}^{+}$scavenging potential among O. wuchuanensis AOPs, demonstrated 20-fold weaker antioxidant activity than that of Andersonin-AOP1 and required a concentration of approximately $60 \mu \mathrm{M}$ to scavenge most of the $\mathrm{ABTS}^{+}$(Fig. 5B). These results indicated that of AOPs from high-altitude $O$. andersonii exhibited more potential antioxidant activities than those of AOPs from cavernicolous $O$. wuchuanensis.

We also compared the scavenging rate of Andesonin-AOP1 and Wuchuannian-AOP5. As shown in Fig. 5C,D, Andersonin-AOP1 reached the maximum scavenging rate in less than $5 \mathrm{~s}$; however, Wuchuanin-AOP5 exhibited a 600 -fold slower scavenging rate and required more than $1800 \mathrm{~s}$ to reach its maximum rate. These results indicated that the free radical scavenging rate of the tested AOP from high-altitude $O$. andersonii was much faster than that of the AOP from cavernicolous $O$. wuchuanensis.

Role of free cysteine in free radical scavenging activities of amphibian skin AOPs. Most AOPs from O. andersonii contained a free cysteine residue, while all AOPs from $O$. wuchuanensis contained no cysteine residue (Fig. 4). Based on this observation, it might be reasonable to speculate that free cysteine plays an important role in the AOPs system of $O$. andersonii. Accordingly, we selected five different AOPs from O. andersonii, Andersonin-AOP19a and -AOP20 (no Cys), -AOP1 and -AOP6 (one Cys) and -AOP12 (two linked Cys) to compare their antioxidant activities. At a concentration of $3 \mu \mathrm{M}$, Andersonin-AOP1 scavenged almost all the $\mathrm{ABTS}^{+}$; however, Andersonin-AOP6 needed a concentration of $50 \mu \mathrm{M}$ to exhibit the same potentiality. When the concentration was lower than $12 \mu \mathrm{M}$, the antioxidant activity of Andersonin-AOP12 was lower than that of -AOP6; however, when the concentration was higher than $12 \mu \mathrm{M}$, the opposite result was found. The same situation also occurred for Anersonin-AOP20. Among these five AOPs, Andersonin-AOP19a was the worst ABTS ${ }^{+}$ scavenger (Fig. 5A). At low concentrations, AOPs with a free cysteine residue showed much stronger antioxidant activities, although the difference was not so obvious at high concentrations. Thus we speculated that the cysteine residue did not determine the antioxidant activities of AOPs and the antioxidant potentiality of specific AOPs did not rely on free cysteine.

We also investigated whether cysteine had an influence on the scavenging rate of AOPs. Andesonin-AOP1 and Andersoin-AOP12 reached their maximum scavenging rates within $5 \mathrm{~s}$ (Fig. 5C,D); however, AOPs with no cysteine residue, including Andersonin-AOP20 and Wuchuanin-AOP5, required approximately $1800 \mathrm{~s}$ to reach their maximum scavenging rates (Fig. 5E,F). These results implied that cysteine played an important role in the scavenging rates of AOPs. To test this speculation, we selected Andersonin-AOP1 as a template and designed four mutants, Andersonin-AOP1-M1 (C7/G7), Andersonin-AOP1-M2 (E6/G6), Andersonin-AOP1-M3 (V8/G8) and Andersonin-AOP1-M4 (W9/G9). As illustrated in Fig. 5G, the replacement of these four residues of Andersonin-AOP1 decreased its free radical scavenging activity and the ninth residue of $\mathrm{W}$ and eighth residue of $\mathrm{V}$ notably decreased the $\mathrm{ABTS}^{+}$scavenging activity. We also investigated the role of these four residues on $\mathrm{ABTS}^{+}$ scavenging efficiency of Andersonin-AOP1. As illustrated in Fig. 5H, -AOP1 scavenged more than 90\% of ABTS ${ }^{+}$ within $5 \mathrm{~s}$, while -M2, -M3 and -M4 achieved maximum scavenging rates within $10 \mathrm{~s}$. However, the efficiency of -M1 was much slower, requiring more than $300 \mathrm{~s}$ to achieve its maximum rate. These results indicated that free cysteine was responsible for the $\mathrm{ABTS}^{+}$scavenging efficiency of -AOP1.

\section{Discussion}

High-elevation environments impose severe physiological challenges on organisms, and elucidating the process and molecular basis of adaptation is a long-standing goal of modern evolutionary biology ${ }^{1,2}$. Despite extensive research, however, knowledge of these crucial issues has been limited to genomic studies of adaptations of endothermic species to the lower oxygen and temperature levels at high elevation, with comparative proteomic analyses, which are essential for understanding how animals adapt to high-elevation environments, still in their infancy. In our previous work, we explored the bioactive peptides of amphibian skins for the development of novel medicines and in the hope of providing meaningful clues on how they adapt to specific surroundings. For example, because bare amphibian skins are vulnerable to pathogens, a variety of antimicrobial peptides are secreted to kill microbes directly ${ }^{31-37}$, and protein weapons have evolved to cope with infection ${ }^{38}$. Following these studies, especially those on the antimicrobial peptides of odorous frogs in which several AOPs were cloned ${ }^{30}$, we chose high-altitude $O$. andersonii and cavernicolous $O$. wuchuanensis for peptidomic investigation to understand the molecular basis for ectothermic species adaptation to UV radiation at high altitude. Although they belong to the same genus (Odorrana, Ranidae), differences in their habitat are remarkable. Odorrana andersonii is widely distributed in the Yun-Gui Plateau in southwestern China, and though its nocturnal lifestyle does limit its exposure to UV radiation, it is still at great risk of direct exposure throughout its lifecycle and during certain activities. Conversely, O. wuchuanensis, which is only distributed in a few mountain caves in Guizhou Province, experiences no UV radiation throughout its entire life cycle (Fig. 1).

The antioxidant activity of $O$. andersonii and $O$. wuchuanensis skin secretions increased slightly when the frogs were maintained in a dark environment without UV radiation (Fig. 2), which may be due to amphibian skins being susceptible to biological or non-biological injuries that induce oxidative stress, such as microorganism infection, parasitization, predation, radiation and aseptic wound $\mathrm{s}^{27,39}$. When mammals are exposed to UVB radiation, for example, an increase in oxidative stress and levels of antioxidant substances, such as SOD and GSH, is often observed ${ }^{40}$. However, how amphibian skin responds when directly exposed to UV radiation remains unclear.

We demonstrated that antioxidant activities were inducible for both selected frogs; however, skin reactions to UV radiation were much stronger in O. andersonii than that in O. wuchuanensis, as evidenced by the differences in their free radical scavenging activities. We also evaluated damage to the skin tissue induced by UV 
radiation. Interestingly, under the same dose of UV radiation, the skin tissue of $O$. andersonii was nearly undamaged, whereas the skin of $O$. wuchuanensis exhibited severe necrosis (Fig. 2), indicating that O. andersonii skin was much more tolerant to high-altitude UV radiation. Because antioxidants usually protect skin from damage induced by UV radiation, we speculated that differences in the antioxidant activities of skin secretions were responsible for the different levels of damage induced by UV radiation.

Skins of $O$. andersonii secreted many more peptides than that of $O$. wuchuanensis when exposed to UV radiation. While no information was available on the structures and functions of these peptides, their molecular weights were less than $4000 \mathrm{Da}$ (Fig. 3 and Table S1) and might function as antioxidants. We identified 46 AOPs from $O$. andersonii, but only nine from $O$. wuchuanensis, which indicated that the diversity of AOPs identified from $O$. andersonii skin secretions was much richer than that of AOPs from O. wuchuanensis skin secretions. Furthermore, antioxidant activities of $O$. andersonii AOPs were more potent than those of $O$. wuchuanensis (Table 1, Fig. 5 and S3-33). These results revealed that $O$. andersonii skin AOPs were more potent and numerous under UV radiation challenge than those of O. wuchuanensis. Thirty-six AOPs were previously identified from the skin secretions of Rana pleuraden, a pond frog widely distributed on the Yun-Gui Plateau ${ }^{28}$. The AOPs from $O$. andersonii and $R$. pleuraden exhibited similar diversities and antioxidant potencies but differed in their primary structures. Thus, powerful AOPs may be a characteristic of amphibians from plateau environments. Although antioxidant enzymes such as SOD increase with altitude in some plant species ${ }^{17}$, their profiles of expression in animals remain scare and to be elucidated.

We found that free cysteine did not impact antioxidant activities, but did have considerable influence on the scavenging rate of AOPs (Fig. 5). Generally speaking, AOPs containing free cysteine exhibited a much faster free radical scavenging rate. In a previous report, free cysteine was proven to be crucial in amphibian AOPs ${ }^{29}$. Most AOPs from O. andersonii skin contained a free cysteine (Fig. 4), this notable and interesting feature also implied that $O$. andersonii skin AOPs were more potent than those of $O$. wuchuanensis.

A potential issue to be considered is that, in addition to the absence of UV exposure, O. wuchuanensis also lives in an obscured environment with high humidity and low temperature. Thus, the molecular basis of amphibian adaptation to high-altitude or cave habitats is likely comprehensive and closely interrelated. This research eliminated these factors by use of a simulated environment and directly demonstrated that AOPs were induced by UV exposure in both frog species. Our results suggested that $O$. andersonii skin evolved a much more complicated and powerful AOPs system. However, we cannot exclude the possibility that these peptides also function in obscured habitats with high humidity and low temperatures, and further research is warranted to characterize the functions of these peptides under different conditions.

\section{Conclusions}

Our results showed that the skins of high-altitude O. andersonii were much more tolerant to UV radiation than that of $O$. wuchuanensis, and demonstrated much richer AOP diversity and greater potency of free racial scavenging activities. These findings indicated that to survive in environments with elevated UV radiation, the skins of $O$. andersonii evolved a much more complicated and powerful antioxidant peptide system. Our results also provided the first peptidomic clues to elucidate the unique molecular basis of adaptation to high altitudes in amphibians.

\section{Materials and Methods}

Animals and ethics statement. Odorrana andersonii specimens were collected in Baoshan City, Yunnan Province, China, and O. wuchuanensis specimens were collected in Wuchuan County, Guizhou Province, China. All study protocols and procedures were approved by the Ethics Committee of the Institutional Review Board of the Kunming Institute of Zoology, Chinese Academy of Sciences, and were conducted in strict accordance with the guidelines for Animal Care and Use at the Kunming Institute of Zoology.

Inducibility of antioxidant activities of skin secretions and skin tolerance to UVB exposure. Once at the laboratory, the odorous frogs were maintained in an artificial pond with food sufficient for one week. Three adult frog specimens of each species were weighed (50-52 g), washed with $\mathrm{ddH}_{2} \mathrm{O}$ and transferred to a plastic box $(13 \times 9 \times 9 \mathrm{~cm})$ filled with $100 \mathrm{ml}$ of $\mathrm{ddH}_{2} \mathrm{O}$ either in the dark (negative control) or in a UVB-exposed environment for $9 \mathrm{~h}$ at room temperature; the seclusion, humidity and other parameters of the artificial environments were identical. The UVB radiation dose was $1600 \mu \mathrm{W} / \mathrm{cm}^{2}$, equivalent to midday in summer in Baoshan city. To confirm the inducibility of the antioxidant activities of the skin secretions, liquid skin secretion samples $(500 \mu \mathrm{l})$ were collected every $1 \mathrm{~h}$ to test their free radical scavenging activities. To compare the extent of damage to skin tissues, the frogs were sacrificed, and the dorsum skins were isolated rapidly for HE staining after UVB radiation exposure.

Collection of skin secretions. Odorrana andersonii and O. wuchuanensis were exposed to UVB at $1600 \mu \mathrm{W} / \mathrm{cm}^{2}$ for $9 \mathrm{~h}$ in a plastic box $(13 \times 9 \times 9 \mathrm{~cm})$ filled with $100 \mathrm{ml}$ of $\mathrm{ddH}_{2} \mathrm{O}$. The collected solutions were centrifuged at $4000 \mathrm{rpm}$ for $15 \mathrm{~min}$, and the supernatants were lyophilized and stored at $-80^{\circ} \mathrm{C}$ until use.

Peptide purification. Peptides were purified in two steps by reverse-phase (RP) chromatography. The dissolved samples were applied to a $\mathrm{C}_{18}$ RP-HPLC column (Hypersil BDS $\mathrm{C}_{18}, 4.0 \times 300 \mathrm{~mm}$, Elite, Dalian, China) pre-equilibrated in $0.1 \%(\mathrm{v} / \mathrm{v})$ trifluoroacetic acid (TFA) in water. The peptides were eluted by a linear gradient $(0-100 \%$ in $100 \mathrm{~min})$ of $0.1 \%(\mathrm{v} / \mathrm{v})$ TFA in acetonitrile $(\mathrm{ACN})$ at a flow rate of $1 \mathrm{ml} / \mathrm{min}$ and monitoring at $215 \mathrm{~nm}$. Peaks of antioxidant activity were collected and lyophilized, and these samples were then further purified on the same $\mathrm{C}_{18}$ column with elution at a more shallow gradient of $0.1 \%$ (v/v) TFA in ACN (0-100\% in $\left.200 \mathrm{~min}\right)$. 
Structural analysis of peptides. The observed molecular weights and purities of the samples were determined using an autoflex speed TOF/TOF mass spectrometer (Bruker Daltonik GmbH, Bremen, Germany) in linear mode with $\alpha$-cyano-4-hydrorycinnamic acid as the matrix. All procedures were conducted according to the manufacturer's standard protocols, and the data were analyzed using the software package provided by the manufacturer.

The complete peptide sequences were determined by Edman degradation on a PPSQ-31A protein sequencer (Shimadzu, Japan) according to the manufacturer's protocols.

Construction of the skin cDNA library. Total RNA was extracted using TRIzol Reagent (Invitrogen, Carlsbad, CA, USA) from the skin of one individual from each frog species. The frogs were washed with deionized water and sacrificed, with the skins immediately stripped, cut into pieces and ground in liquid nitrogen. We purified the mRNA using an Absolutely mRNA Purification Kit according to standard protocols (Stratagene, Canada). The cDNA was synthesized using SMART ${ }^{\mathrm{TM}}$ techniques with a SMART cDNA Library Construction Kit (Clontech, Canada). The first strand of cDNA was synthesized using 3' SMART CDS III/3' PCR primer, SMART IV oligonucleotide and SMART ${ }^{\mathrm{TM}}$ MMLV Reverse Transcriptase (Clontech, Canada). The second strand was amplified using Advantage polymerase with 5' PCR primer and CDS III/3' PCR primer according to the protocols of the kit.

Screening of cDNAs encoding AOP precursors. The synthesized cDNAs were used as templates for high-stringency PCR amplification to screen for cDNAs encoding AOP precursors. Two primers, S1 (5'-CCAAA(G/C)ATGTTCACC(T/A)TGAAGAAA-3') and different 3' PCR primers designed according to the peptide sequences provided by Edman degradation were used in the PCR reactions. Advantage polymerase was used in the reactions. The PCR procedure was as follows: $2 \mathrm{~min}$ at $94^{\circ} \mathrm{C}$, followed by 30 cycles of $10 \mathrm{~s}$ at $92^{\circ} \mathrm{C}$, $30 \mathrm{~s}$ at $50^{\circ} \mathrm{C}$ and $40 \mathrm{~s}$ at $72^{\circ} \mathrm{C}$. The PCR products were then recovered using a DNA Gel Extraction Kit (Bioteke, China) and ligated into the vector pMD19-T (Takara Biotechnology, China). Finally, the PCR products were cloned into competent Escherichia coli DH5 $\alpha$. Clones were randomly selected for DNA sequencing on an Applied Biosystems DNA sequencer (ABI 3730XL, USA).

Peptide synthesis. All peptides used in the evaluations of bioactivity were synthesized by solid-phase synthesis on an Applied Biosystems model 433A peptide synthesizer according to standard protocols, as previously reported $^{32}$. Purities of the synthetic peptides were greater than $95 \%$.

Free radical scavenging activity assay. Two free radical scavenging tests were adopted to evaluate the antioxidant activity of the samples.

A 2, 2'-azino-bis (3-ethylbenzothiazoline-6-sulfonic acid) (ABTS) scavenging test was performed as previously described ${ }^{41}$, with some modification. Briefly, a stock solution of ABTS radical (Sigma-Aldrich, USA) was prepared by incubating $2.8 \mathrm{mM}$ potassium persulfate (Sigma-Aldrich, USA) with $7 \mathrm{mM}$ ABTS in water for at least $6 \mathrm{~h}$ in the dark, after which it was used immediately. The stock solution was diluted 50 -fold with $\mathrm{ddH}_{2} \mathrm{O}$. The samples dissolved in water were added, and the same volume of solvent was used as a negative control. Vitamin $\mathrm{C}$ dissolved in $\mathrm{H}_{2} \mathrm{O}$ was used as the positive control. The reaction was kept from light for $10 \mathrm{~min}$. The decrease in absorbance at $415 \mathrm{~nm}$ indicated the antioxidant activity of the samples. The rate of free radical scavenging (\%) was calculated by $\left(\mathrm{A}_{\text {blank }}-\mathrm{A}_{\text {sample }}\right) \times 100 / \mathrm{A}_{\text {blank }}$.

A 2, 2-diphenyl-1-picrylhydrazyl (DPPH) scavenging test was also performed as previously described ${ }^{42}$, with some modification. Briefly, the assay mixture contained $190 \mu \mathrm{l}$ of $5 \times 10^{-5} \mathrm{M}$ DPPH radical (Sigma-Aldrich, St Louis, MO, USA) dissolved in methanol. The sample solution $(10 \mu \mathrm{l})$ was then incubated in a sealed 1.5 - $\mathrm{ml}$ microcentrifuge tube for $30 \mathrm{~min}$ at room temperature, and the absorbance was read against a blank at $517 \mathrm{~nm}$. The DPPH scavenging activity $(\%)$ was calculated by $\left(\mathrm{A}_{\text {blank }}-\mathrm{A}_{\text {sample }}\right) \times 100 / \mathrm{A}_{\text {blank }}$.

Hematoxylin-eosin (HE) staining. Isolated frog skin tissues were fixed in $10 \%$ buffered formalin overnight and then immersed in 70\% ethanol for storage at room temperature. The tissues were embedded in paraffin. Sections with a thickness of $5 \mu \mathrm{m}$ were cut on positively charged slides. The sections were stained with HE solution. All tissue samples were examined and photographed in a blinded manner. Images were captured using an Olympus BX51 microscope (Olympus, Tokyo, Japan) at $\times 40$ or $\times 100$ magnification $^{43}$.

\section{References}

1. Smith, N. G. \& Eyre-Walker, A. Adaptive protein evolution in Drosophila. Nature 415, 1022-1024 (2002).

2. McDonald, J. H. \& Kreitman, M. Adaptive protein evolution at the Adh locus in Drosophila. Nature 351, 652-654 (1991).

3. Blumthaler, M., Ambach, W. \& Ellinger, R. Increase in solar UV radiation with altitude. J Photoch Photobio B 39, 130-134 (1997).

4. Bickler, P. E. \& Buck, L. T. Hypoxia tolerance in reptiles, amphibians, and fishes: Life with variable oxygen availability. Annu Rev Physiol 69, 145-170 (2007).

5. Storz, J. F. et al. The molecular basis of high-altitude adaptation in deer mice. PLoS genetics 3, 448-459 (2007).

6. Weber, R. E. High-altitude adaptations in vertebrate hemoglobins. Resp Physiol Neurobi 158, 132-142 (2007).

7. Yu, L., Wang, X. P., Ting, N. \& Zhang, Y. P. Mitogenomic analysis of Chinese snub-nosed monkeys: Evidence of positive selection in NADH dehydrogenase genes in high-altitude adaptation. Mitochondrion 11, 497-503 (2011).

8. Qiu, Q. et al. The yak genome and adaptation to life at high altitude. Nature genetics 44, 946-949 (2012).

9. Ge, R. L. et al. Draft genome sequence of the Tibetan antelope. Nature communications 4, 1858 (2013).

10. Bigham, A. et al. Identifying signatures of natural selection in Tibetan and Andean populations using dense genome scan data. PLoS genetics 6, e1001116 (2010).

11. Simonson, T. S. et al. Genetic evidence for high-altitude adaptation in Tibet. Science 329, $72-75$ (2010).

12. Cho, Y. S. et al. The tiger genome and comparative analysis with lion and snow leopard genomes. Nature communications $\mathbf{4}, 2433$ (2013). 
13. Li, M. et al. Genomic analyses identify distinct patterns of selection in domesticated pigs and Tibetan wild boars. Nature genetics 45 , 1431-1438 (2013).

14. Bigham, A. W. et al. Identifying positive selection candidate loci for high-altitude adaptation in Andean populations. Human genomics 4, 79-90 (2009).

15. Alkorta-Aranburu, G. et al. The genetic architecture of adaptations to high altitude in Ethiopia. PLoS genetics 8, e1003110 (2012).

16. Scheinfeldt, L. B. et al. Genetic adaptation to high altitude in the Ethiopian highlands. Genome biology 13, R1 (2012).

17. Ma, L. et al. Physiological, biochemical and proteomics analysis reveals the adaptation strategies of the alpine plant Potentilla saundersiana at altitude gradient of the Northwestern Tibetan Plateau. Journal of proteomics 112C, 63-82 (2014).

18. Muir, A. P., Biek, R. \& Mable, B. K. Behavioural and physiological adaptations to low-temperature environments in the common frog, Rana temporaria. Bmc Evol Biol 14 (2014).

19. Navas, C. A. \& Chaui-Berlinck, J. G. Respiratory physiology of high-altitude anurans: 55 years of research on altitude and oxygen. Resp Physiol Neurobi 158, 307-313 (2007).

20. Hou, P. C. L. \& Huang, S. P. Metabolic and ventilatory responses to hypoxia in two altitudinal populations of the toad, Bufo bankorensis. Comp Biochem Phys A 124, 413-421 (1999).

21. Biswas, H. M., Patra, P. B. \& Boral, M. C. Body-Fluid and Hematologic Changes in the Toad Exposed to 48 H of Simulated HighAltitude. J Appl Physiol 51, 794-797 (1981).

22. Weber, R. E. et al. Novel mechanism for high-altitude adaptation in hemoglobin of the Andean frog Telmatobius peruvianus. Am J Physiol-Reg I 283, R1052-R1060 (2002).

23. Yang, W., Qi, Y., Bi, K. \& Fu, J. Toward understanding the genetic basis of adaptation to high-elevation life in poikilothermic species: a comparative transcriptomic analysis of two ranid frogs, Rana chensinensis and R. kukunoris. BMC genomics 13, 588 (2012).

24. Kohen, R. \& Gati, I. Skin low molecular weight antioxidants and their role in aging and in oxidative stress. Toxicology 148, 149-157 (2000).

25. Shindo, Y. et al. Antioxidant Defense-Mechanisms in Murine Epidermis and Dermis and Their Response to Ultraviolet-Light. $J$ Invest Dermatol 100, 600-600 (1993).

26. Kohen, R. Skin antioxidants: their role in aging and in oxidative stress - New approaches for their evaluation. Biomed Pharmacother 53, 181-192 (1999).

27. Clarke, B. T. The natural history of amphibian skin secretions, their normal functioning and potential medical applications. Biol Rev 72, 365-379 (1997).

28. Yang, H. et al. Antioxidant peptidomics reveals novel skin antioxidant system. Molecular \& cellular proteomics: MCP 8, 571-583 (2009).

29. Liu, C. B. et al. Frog skins keep redox homeostasis by antioxidant peptides with rapid radical scavenging ability. Free Radical Bio Med 48, 1173-1181 (2010).

30. Yang, X., Lee, W. H. \& Zhang, Y. Extremely abundant antimicrobial peptides existed in the skins of nine kinds of Chinese odorous frogs. Journal of proteome research 11, 306-319 (2012).

31. Lee, W. H. et al. Variety of antimicrobial peptides in the Bombina maxima toad and evidence of their rapid diversification. Eur J Immunol 35, 1220-1229 (2005)

32. Lee, W. H. et al. Maximin 9, a novel free thiol containing antimicrobial peptide with antimycoplasma activity from frog Bombina maxima. FEBS letters 579, 4443-4448 (2005).

33. Zhang, Y. Why do we study animal toxins? Dong wu xue yan jiu = Zoological research / "Dong wu xue yan jiu" bian ji wei yuan hui bian ji 36, 183-222 (2015).

34. Lai, R., Liu, H., Hui Lee, W. \& Zhang, Y. An anionic antimicrobial peptide from toad Bombina maxima. Biochemical and biophysical research communications $295,796-799$ (2002).

35. Wang, T. et al. Maximins S, a novel group of antimicrobial peptides from toad Bombina maxima. Biochemical and biophysical research communications 327, 945-951 (2005).

36. Lee, W. H. et al. Maximin 9, a novel free thiol containing antimicrobial peptide with antimycoplasma activity from frog Bombina maxima. FEBS letters 579, 4443-4448 (2005).

37. Chen, Z. M. et al. Two novel families of antimicrobial peptides from skin secretions of the Chinese torrent frog, Amolops jingdongensis. Biochimie 94, 328-334 (2012).

38. Xiang, Y. et al. Host-derived, pore-forming toxin-like protein and trefoil factor complex protects the host against microbial infection. Proceedings of the National Academy of Sciences of the United States of America 111, 6702-6707 (2014).

39. Zasloff, M. Antimicrobial peptides of multicellular organisms. Nature 415, 389-395 (2002).

40. Svobodova, A. R. et al. Acute exposure to solar simulated ultraviolet radiation affects oxidative stress-related biomarkers in skin, liver and blood of hairless mice. Biological \& pharmaceutical bulletin 34, 471-479 (2011).

41. Akerstrom, B., Maghzal, G. J., Winterbourn, C. C. \& Kettle, A. J. The lipocalin alpha1-microglobulin has radical scavenging activity. The Journal of biological chemistry 282, 31493-31503 (2007).

42. Wen, K. C. et al. Antioxidant activity of Ixora parviflora in a cell/cell-free system and in UV-exposed human fibroblasts. Molecules 16, 5735-5752 (2011)

43. Yin, Y. et al. Quercitrin protects skin from UVB-induced oxidative damage. Toxicology and applied pharmacology 269, 89-99 (2013).

\section{Acknowledgements}

This work was supported by grants from the Chinese National Natural Science Foundation (U1132601, 31270835, 31572268 and 31460571) and the Key Research Program of the Chinese Academy of Sciences (KJZD-EW-L03).

\section{Author Contributions}

Y.Z. and X.W.Y. designed the study. X.W.Y. and Y.W. performed most of the research, including the collection of animals, purification procedures, determination of activity and primary structures of peptides, HE staining and preparation of the manuscript. Y.Z. and W.H.L. participated in the purification procedure and antioxidant activity determination of peptides. All authors contributed substantially to this research and reviewed this manuscript.

\section{Additional Information}

Supplementary information accompanies this paper at http://www.nature.com/srep

Competing financial interests: The authors declare no competing financial interests.

How to cite this article: Yang, X. et al. Rich diversity and potency of skin antioxidant peptides revealed a novel molecular basis for high-altitude adaptation of amphibians. Sci. Rep. 6, 19866; doi: 10.1038/srep19866 (2016).

This work is licensed under a Creative Commons Attribution 4.0 International License. The images or other third party material in this article are included in the article's Creative Commons license, unless indicated otherwise in the credit line; if the material is not included under the Creative Commons license, users will need to obtain permission from the license holder to reproduce the material. To view a copy of this license, visit http://creativecommons.org/licenses/by/4.0/ 